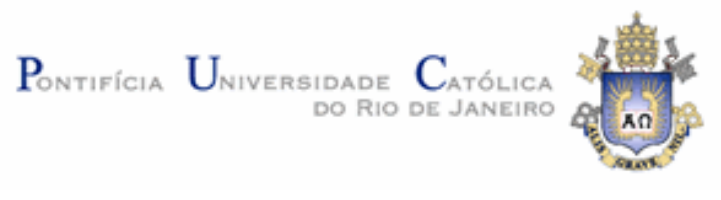

Marcela Lobo Francisco

\title{
Uma comparação entre os regimes de taxação sobre o petróleo: concessão e partilha
}

\begin{abstract}
Tese de Doutorado
Tese apresentada como requisito parcial para obtenção do título de Doutor pelo Programa de PósGraduação em Engenharia de Produção da PUCRio.
\end{abstract}

Orientador: José Paulo Teixeira

Rio de Janeiro

Agosto de 2011 


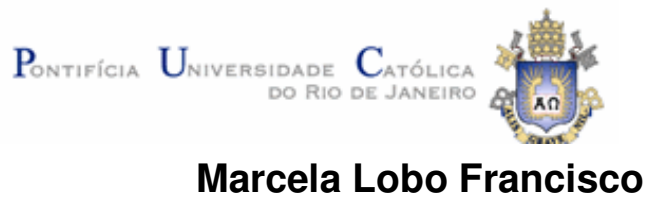

\section{Uma comparação entre os regimes de taxação sobre o petróleo: concessão e partilha}

Tese apresentada como requisito parcial para obtenção do título de Doutor pelo Programa de Pós-Graduação em Engenharia de Produção da PUC-Rio. Aprovada pela Comissão Examinadora abaixo assinada.

Prof. José Paulo Teixeira Orientador PUC-Rio

Prof. José Paulo Teixeira PUC-Rio

Prof. Katia Maria Carlos Rocha IPEA, PUC-Rio

Prof.- Marco Antônio Guimarães Dias Petrobras, PUC-Rio

Prof. - Lucia Helena Salgado Instituto de Pesquisa Econômica e Aplicada, IPEA

Prof.- Maria Bernadete Gomes Pereira Sarmiento Gutierrez Instituto de Pesquisa Econômica e Aplicada, IPEA

Prof.- José Eugenio Leal

Coordenador(a) Setorial do Centro Técnico Científico - PUC-Rio 
Todos os direitos reservados. É proibida reprodução total ou parcial do trabalho se autorização da universidade, da autora e do orientador.

\section{Marcela Lobo Francisco}

Possui graduação em Estatística pela Universidade do Estado do Rio de Janeiro e em Ciências Econômicas pela Universidade Federal do Rio de Janeiro e é mestre em Engenharia Industrial pela Pontifícia Universidade Católica do Rio de Janeiro. Participou do projeto PUC/Petrobras de análise de investimentos usando a teoria das opções reais da planta GTL e é professora da Universidade Gama Filho.

Ficha Catalográfica

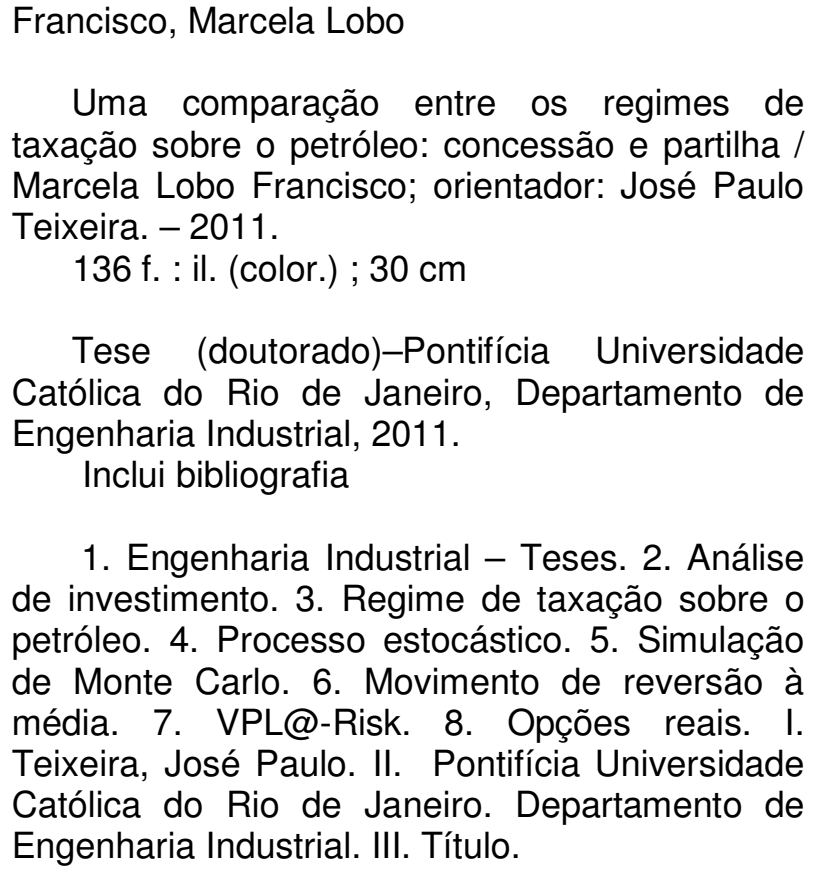

1. Engenharia Industrial - Teses. 2. Análise de investimento. 3. Regime de taxação sobre o petróleo. 4. Processo estocástico. 5. Simulação de Monte Carlo. 6. Movimento de reversão à média. 7. VPL@-Risk. 8. Opções reais. I. Teixeira, José Paulo. II. Pontifícia Universidade Católica do Rio de Janeiro. Departamento de Engenharia Industrial. III. Título.

CDD: 658.5 
As minhas irmãs, Daniela e Luciana, e ao meu cunhado Breno, que ao longo de todo o caminho percorrido sempre estiveram ao meu lado.

A minha mãe, mulher de extrema coragem e força, na qual eu me inspiro e procuro seguir os passos, sem a qual eu não teria a força necessária para mais uma vez seguir em frente e me manter de pé.

Ao meu pai, homem de extrema inteligência e capacidade, no qual eu me inspiro e procuro seguir os passos, sem o qual eu não teria a sabedoria necessária para mais uma vez seguir em frente e me manter de pé.

Aos meus pais que nunca desistiram de mim. 


\section{Agradecimentos}

Ao professor José Paulo Teixeira, meu orientador no mestrado e doutorado. Que sempre me ajudou no desenvolvimento do meu trabalho.

A professora Katia Rocha pela disponibilidade nos momentos de dúvida, e pela atenção nos momentos de dificuldade.

As professoras Lucia Helena e Maria Bernadete e ao professor Marco Antônio, pela disponibilidade em participar da banca.

A Claudia que sempre se mostrou solicita nas inúmeras dúvidas acerca dos procedimentos necessários ao longo do curso de Doutorado.

A Coordenação de Aperfeiçoamento de Pessoal de Nível Superior (CAPES) pelo apoio financeiro.

Por fim, a Pontifícia Universidade Católica por todo conhecimento transmitido.

\section{"Ensinar exige segurança, competência profissional e generosidade". Paulo Freire}




\section{Resumo}

Francisco, Marcela Lobo; Teixeira, José Paulo. Uma comparação entre os regimes de taxação sobre o petróleo: concessão e partilha. Rio de Janeiro, 2011. 136p. Tese de Doutorado - Departamento de Engenharia Industrial, Pontifícia Universidade Católica do Rio de Janeiro.

A escolha, por parte dos governos, de qual regime de taxação adotar sobre o petróleo determina como a renda gerada pela atividade de exploração e produção deste é dividida entre o Estado e os investidores particulares. Por parte dos Estados é extremamente importante a escolha de um regime que não desestimule os investidores particulares, e que por outro lado seja capaz de gerar renda suficiente para impulsionar a sua economia e produzir o bem estar da sua população. Este trabalho faz um estudo dos principais regimes de taxação sobre o petróleo identificando como a renda gerada pelo campo é distribuída entre o governo e os investidores particulares, e calcula a exposição ao risco dos agentes para ambos os regimes estudados. O objetivo é verificar se estes atendem aos principais requisitos que um regime deve ter para ser considerado eficiente (simplicidade e neutralidade) e se existe uma relação entre o tipo de regime e as variáveis citadas acima. Os principais regimes de taxação existentes no mundo são o regime de concessão e o regime de partilha. Foram estudados os regimes vigentes na Austrália, na Noruega, no Brasil e na Indonésia. Os três primeiros países adotam o regime de concessão e o último adota o regime de partilha. Dentre os regimes estudados o da Austrália mostrou ser o mais simples, sendo a remuneração do governo feita através de duas taxas, e o da Indonésia o mais complexo, onde a remuneração do governo é feita através de cinco taxas. O Brasil apresenta um regime considerado simples, sendo a receita do governo feita através de três taxas. A distribuição da renda gerada pela atividade de exploração e produção do petróleo é mais equitativa na Austrália onde os investidores particulares recebem cerca de $16,6 \%$ e o governo $88,4 \%$. No Brasil cabe aos investidores particulares $9,16 \%$ e ao governo 90,84\%. A Noruega e a Indonésia apresentaram regimes de taxação que penalizam o investidor particular, o campo apresenta um VPL positivo antes do pagamento das taxas e negativo após. O 
Brasil e a Austrália apresentaram VPL positivo antes e depois do pagamento das taxas. Através dos resultados encontrados pode-se verificar que não existe uma relação entre o tipo de regime e as seguintes variáveis: equidade na distribuição da renda, neutralidade e exposição ao risco do investidor particular e do governo. Países que adotam o mesmo regime, de concessão, apresentaram características diferentes: Noruega mostrou ser um regime que penaliza o investidor particular e a Austrália um que possui a distribuição de renda mais equitativa. Em relação ao risco o que apresentou um maior risco para o investidor particular foi a Indonésia e o que apresentou menor risco foi a Austrália. O Brasil em ambos os casos ficou em terceiro lugar. Sendo assim, um regime em si não pode ser considerado mais eficiente do que outro. A questão principal é a forma como a taxação é feita dentro de cada regime. O foco da discussão deveria ser como o petróleo vai ser taxado dentro do atual regime (concessão), dado que agora o volume de reservas do país atingiu um nível inédito, e não qual o regime de taxação adotar.

\section{Palavras-chave}

Análise de investimento; regime de taxação sobre o petróleo; processo estocástico; simulação de Monte Carlo; movimento de reversão à média; VPL@ Risk; Opções Reais. 


\section{Abstract}

Francisco, Marcela Lobo; Teixeira, José Paulo (Advisor). Comparison between petroleum taxation: concessionary and sharing. Rio de Janeiro, 2011. 136p. - DSc. Thesis - Departamento de Engenharia Industrial, Pontifícia Universidade Católica do Rio de Janeiro.

Government choice of which taxation regime to adopt on oil determines how the revenue generated by the activity of exploring and producing this oil is shared between the State and private investors. From the point of view of the State, it is extremely important to choose a regime that does not detract private investors and that is also capable of generating enough revenue to bolster the country's economy and promote the well-being of its population. The present work studies the main taxation regimes on oil, identifying how the income generated by this product is distributed between government and private investors. It also calculates the level of risk for agents in both regimes analysed. The aim is to verify if these regimes comply with the main requirements necessary for a regime to be considered efficient - simplicity and neutrality - and if there is a relation between the kind of regime and the variables mentioned above.The main taxation regimes in the world are concession and sharing. The regimes adopted by Australia, Norway, Brazil and Indonesia were studied. The first three countries adopt the concession regime, while the latter adopts the sharing one. The Australian regime - in which government remuneration was obtained by two taxes - was the simplest of all analysed regimes, while that of Indonesia - in which government remuneration was obtained by five taxes - was the most complex. The Brazilian regime is considered to be simple: government revenue is obtained by means of three taxes. The distribution of the revenue generated by oil exploration and production is more balanced in Australia, where private investors receive around $16.6 \%$ and the government around $88.4 \%$. In Brazil private investors gain $9.16 \%$ and the government $90.84 \%$.The regimes in Norway and Indonesia penalize the private investor; the field presents a positive net current value prior to taxes and negative after taxes. Brazil and Australia displayed positive net current value prior to and after taxes levied. The findings suggest that 
there is no relation between the kind of regime and the following variables: equity in income distribution, neutrality, and investor and government risk. Countries that adopt the same regime - concession - presented different characteristics. The regime adopted by Norway penalized the private investor, while that of Australia distributed revenues more equitably. As regards risk, the regime with greatest risks for private investors was the Indonesian, while the one with least risks was the Australian. Brazil ranked third in both categories. Thus, a regime cannot be considered more efficient than another. The main issue is how taxes are levied within each regime. Given that the Brazilian oil reserve has reached unprecedented levels, discussion should focus on how oil will be taxed in the present regime (concession), rather than on the taxation regime adopted.

\section{Keywords}

Investment analysis; petroleum taxation; stochastic processes; Monte Carlo simulation; mean reversion model; VPL@ Risk; Real Option. 


\section{Sumário}

1 Introdução 16

2 Revisão Bibliográfica $\quad 20$

3 Estado da arte $\quad 24$

4 Origem do petróleo no Brasil 26

4.1. O pré-sal brasileiro 32

5 Regimes de taxação do petróleo 40

5.1. Características de um regime de taxação 41

5.2. Regimes de taxação 44

5.2.1. Regime de concessão 44

$\begin{array}{ll}\text { 5.2.2. Bônus de assinatura } & 47\end{array}$

$\begin{array}{ll}\text { 5.2.3. Royalties } & 48\end{array}$

5.2.4. Participação especial 49

5.2.5. Pagamento pela ocupação ou retenção da área 50

5.3. Partilha da produção 51

6 Processos Estocásticos $\quad 54$

6.1. Principais Processos Estocásticos 55

6.1.1. Processo de Markov 55

6.1.2. Random Walk 56

6.1.3. Processo Autoregressivo de Primeira Ordem (AR 1) 57

6.1.4. Processo de Wiener 58

6.1.5. Movimento Browniano com Drift ou Movimento de Wiener generalizado (Movimento Aritmético Browniano) 59

6.1.6. Movimento Browniano Generalizado - o Processo de Ito 59

6.1.7. Movimento Geométrico Browniano (MGB) 60

6.1.8. Processo de Reversão à Média 63

6.2. Simulação de Monte Carlo do preço do petróleo 65

7 Análise de risco $\quad 72$

$\begin{array}{ll}\text { 7.1. Técnicas de análise de investimento } & 73\end{array}$ 
7.1.1. Valor Presente Líquido (VPL) 73

7.1.2. Taxa Interna de Retorno $\quad 74$

7.1.3. Payback descontado 75

7.2. Características do modelo 76

7.3. Austrália 78

7.4. Noruega $\quad 79$

7.5. Indonésia $\quad 81$

7.6. Fluxo de Caixa no regime de Concessão 83

7.6.1. Fluxo de caixa no regime fiscal da Austrália 84

7.6.2. Fluxo de caixa no regime fiscal da Noruega 86

7.6.3. Fluxo de caixa no regime fiscal do Brasil 89

7.7. Fluxo de caixa no regime de Partilha 92

7.7.1. Fluxo de caixa no regime da Indonésia 93

7.8. Remuneração do governo $X$ dos agentes particulares 101

7.9. Risco X Retorno 104

7.10. Novas regas de taxação para: Noruega, Brasil, Indonésia 113

8 Análise utilizando a teoria das opções reais 117

8.1. As Opções Reais e o Valor da Flexibilidade Gerencial 119

8.2. Opção de adiar o investimento 120

9 Conclusão 126

10 Referências Bibliográficas $\quad 130$

Apêndice A Tabelas para o cálculo da PE 134

Apêndice B Análise do preço 136 


\section{Lista de figuras}

Figura 4.1: Plataforma elevatória P-1 27

Figura 4.2: Roncador: exploração de petróleo em alto mar, 200028

Figura 4.3: Gráfico da razão RP / P 32

Figura 4.4: Pré-Sal no Brasil 33

Figura 4.5: Área do Pré-Sal 34

Figura 4.6: Previsão de barris de petróleo nos campos do Pré-Sal 35

Figura 4.7: Descoberta de novos campos do Pré-Sal 35

Figura 4.8: Evolução da exploração de petróleo pela Petrobras 35

Figura 4.9: Desafios da exploração do pré-sal 36

Figura 4.10: Evolução dos gastos com extração 38

Figura 5.1: Gráfico do valor do bônus de assinatura por rodada de licitação $\quad 48$

Figura 5.2: Gráfico do valor dos royalties de 1998/2009 49

Figura 5.3: Gráfico do valor das participações especiais de 1998/2009 50

Figura 6.1: Gráfico da variância no Movimento Geométrico Browniano 63

Figura 6.2: Gráfico da variância no Movimento de Reversão a Média 65

Figura 6.3: Ilustração da SMC 66

Figura 6.4: Preço do Brent (U\$/bbl)_ 1982/2009 68

Figura 6.5: Série real e nominal do Brent_1982/2009 69

Figura 7.1.Regra de decisão do VPL $\quad 75$

Figura 7.2: The Ekofisk field_Noruega 80

Figura 7.3: Reservas de petróleo_Indonésia $\quad 82$

Figura 7.4: VPL Austrália_4,0_U\$ 54,36 85

Figura 7.5: VPL Austrália_4,0_U\$ 95,00 85

Figura 7.6: VPL Austrália_1,0_U\$ 54,36 85

Figura 7.7: VPL Austrália_1,0_U\$ 95,00 86

Figura 7.8: VPL Noruega_4,0_U\$ 54,36 87

Figura 7.9: VPL Noruega_4,0_U\$ 95,00 88

Figura 7.10: VPL Noruega_1,0_U\$ 54,36 88

Figura 7.11: VPL Noruega_1,0_U\$ 95,00 89

Figura 7.12: VPL Brasil_4,0_U\$ 54,36 90 
Figura 7.13: VPL Brasil_4,0_U\$ 95,00 91

Figura 7.14: VPL Brasil_1,0_U\$ 54,36 91

Figura 7.15: VPL Brasil_1,0_U\$ 95,00 92

Figura 7.16: VPL Indonésia_4,0_U\$ 54,36 94

Figura 7.17: VPL Indonésia_1,0_U\$ 54,36 95

Figura 7.18: VPL Indonésia_1,0_U\$ 95,00 95

Figura 7.19: VPL Indonésia_4,0_U\$ 95,00 96

Figura 7.20: Austrália_VPL X RTG_4,0_U\$ 95,00 108

Figura 7.21: Indonésia_VPL X RTG_4,0_U\$ 95,00 108

Figura 8.1: Quando a flexibilidade gerencial é valiosa 120

Figura 8.2: VP_Fx_Cx - Brasil, Indonésia_U\$ 55,00 121

Figura 8.3: Payoff da opção_Brasil - U\$ 55,00 123

Figura 8.4: VP_Fx_Cx - Brasil, Indonésia_U\$ 90,00 124

Figura 8.5: Payoff da opção_Brasil, Indonésia - U\$ 80,00 125 


\section{Lista de tabelas}

Tabela 4.1: Volume de produção de petróleo (bbl/dia) por país 29

Tabela 4.2: Volume de reservas de petróleo provadas por país 30

Tabela 4.3: Produção de petróleo no país (2000/2009) 31

Tabela 4.4: Produção de petróleo no país (2000/2009) 31

Tabela 4.5: Produção X Custo de extração. 38

Tabela 5.1: Cálculo da alíquota da Participação Especial ( $1^{\circ}$ ano de produção) 49

Tabela 5.2: Vantagens e desvantagens dos regimes de taxação do petróleo 53

Tabela 6.1: Parâmetros dos processos estocásticos (Série Brent: 1982/2009) 69

Tabela 6.2: Parâmetros dos processos estocásticos (Série Brent: 1900/2009) 70

Tabela 6.3: Valores de negociação do Brent no mercado Futuro na NYMEX 71

Tabela 6.4: Valores de negociação do Brent no mercado Futuro na ICE 71

Tabela 7.1: Características do modelo 76

Tabela 7.2: Convergência dos VPLs - média de longo prazo de U\$ 54,36/bbl 77

Tabela 7.3: Convergência dos VPLs - média de longo prazo de U\$ 95,00/bbl 77

Tabela 7.4: Sistemas de Concessão $\quad 81$

Tabela 7.5: VPL_Austrália $\quad 86$

Tabela 7.6: VPL_Noruega $\quad 89$

Tabela 7.7: VPL_Brasil 92

Tabela 7.8: VPL_Indonésia 96

Tabela 7.9: VPLs_U\$54,36/bbl 97

Tabela 7.10: VPLs_U\$95,00/bbl 98

Tabela7.11: Break even point investimento_4,0_U\$ 56,34 99

Tabela 7.12: Break even point investimento_1,0_U\$ 95,00 100

Tabela 7.13: Break even point investimento_4,0_U\$ 95,00 100

Tabela 7.14: Remuneração do governo 101

Tabela 7.15: Remuneração do governo na Austrália 102

Tabela 7.16: Remuneração do governo na Noruega 102

Tabela 7.17: Remuneração do governo no Brasil 103

Tabela 7.18: Remuneração do governo na Indonésia 103

Tabela 7.19: VPL @Risk_4,0_U\$95,00 105 
Tabela 7.20: Coeficiente de variação x (VPL @ Risk/Média)_4,0_U\$95,00 105

Tabela 7.21: RTG @_Risk_4,0 106

Tabela 7.22: Coeficiente de variação x (RTG @Risk/Média)_4,0_U\$95,00 107

Tabela 7.23: Exposição ao risco 107

Tabela 7.24: Participação da remuneração do governo_1,0_U\$95,00/bbl 109

Tabela 7.25: Participação da remuneração do governo_4,0_U\$95,00/bbl 110

Tabela 7.26: VPL sem e com taxas_1,0_U\$95,00/bbl 110

Tabela 7.27: VPL sem e com taxas_4,0_U\$95,00/bbl 111

Tabela 7.28: VPL nova taxação_4,0_U\$95,00/bbl 114

Tabela 7.29: CV x (VPL @ Risk/Média)_Novas regras_4,0_U\$95,00 115

Tabela 7.30: RTG nova taxação_4,0_U\$95,00/bbl 115

Tabela 7.31: CV x (RTG @Risk/Média)_Novas regras_4,0_U\$95,00 115

Tabela 7.32: Distribuição da renda_Novas regras_4,0_U\$95,00 116

Tabela 8.1: Opção Real x Opção Financeira 118

Tabela A.1: Pagamento da PE no segundo ano de produção 134

Tabela A.2: Pagamento da PE no terceiro ano de produção 134

Tabela A.3: Pagamento da PE após o terceiro ano de produção 135

Tabela B.1: Análise do preço 136 